\title{
SUPPRESSOR GENE ACTION IN THE TRYPTOPHAN SYNTHETASE SYSTEM OF NEUROSPORA CRASSA. II. BIOCHEMICAL STUDIES ${ }^{1}$
}

\author{
JOSEPH D. YOURNO 2,3 AND SIGMUND R. SUSKIND \\ McCollum-Pratt Institute, Johns Hopkins University, Baltimore, Maryland
}

Received May 11, 1964

SUPPRESSOR gene action in some cases may be explained by an effect on the primary structure of a mutant protein. This may entail the insertion of the wild type or a different but functional amino acid at the primary mutant site, or another amino acid substitution at a second site, thereby producing a functional double mutant enzyme. The existence of this type of suppressor mechanism was suggested from experiments with one suppressed tryptophan synthetase mutant of Neurospora crassa (Yanofsky 1952). The formation of enzyme which is normal in catalytic, immunological, and physical properties has been shown for a group of suppressed CRM-negative alkaline phosphatase mutants of Escherichia coli (GAREN and Sidprol 1962). (CRM: crossreacting material.) A small amount of wild-type-like enzyme in addition to the mutant species was found in a suppressed CRM-positive tryptophan synthetase A protein mutant of $E$. coli (YANOFSKY and CRAwFord 1959), and recently it was demonstrated that the wild-typelike A protein contained the original wild-type amino acid reinserted in its correct position in the polypeptide chain (BRoDY and YanOFsky 1963).

This paper is concerned with the nature of the tryptophan synthetase protein possessing wild-type activity which has been restored in suppressed $t d_{201}$ mutants as well as with the negligible effect of the $t d_{201}$ suppressors on the wild-type phenotype. The results are considered in the context of the existing hypotheses of suppressor-gene action. A preliminary report of part of this material has been presented (Yourno and Suskind 1963).

\section{MATERIALS AND METHODS}

Growth analyses: Quantitative experiments were performed according to the method of RyAN (1950). For a comparison of the relative growth rates of each unsuppressed wild type $(t d+$ $\left.s u{ }_{201}\right)$ or suppressed wild-type $\left(t d+s u_{201}\right)$ strain on various media, inocula were taken from an identical conidial suspension and still cultures were run concurrently. In direct comparisons of relative growth rates of $t d+s u{ }^{+}{ }_{201}$ and $t d+s u_{201}$ strains on minimal medium, conidial suspensions were filtered once through cheesecloth and counted with a Petroff-Hauser counter. Suspensions then were adjusted to equal conidial concentration. Viable conidial counts were made by plating aliquots of the suspension on sorbose agar. These comparisons were made between

\footnotetext{
1 This research was supported by Public Health Service Grant CA-03080. Contribution No. 424 of the McCollumPratt Institute.

${ }^{2}$ Public Health Service Predoctoral Fellow. This work has been presented in partial fulfillment of the requirements for the degree of Doctor of Philosophy.

${ }^{3}$ Present address: Max Planck Institut für Biologie, Tubingen, Germany. 
$t d{ }^{s u}{ }_{201}$ and $t d+s u_{201}$ strains originating in the same tetrad to decrease the possibility of growth rate differences due to diverse genetic backgrounds. Inocula for all $t d+s u_{201}$ cultures were taken directly from ascospore-initiated cultures to insure the presence of the suppressor gene.

Preparation of extracts: Small quantities of crude extract for comparative assays were prepared as described by Yourno and Suskrnd (1964). Large quantities of mycelia necessary for enzyme purification were grown in 10 or 20 liter carboy culture at $30^{\circ} \mathrm{C}$ as described (MoHLER and Suskind 1960). Wild types were grown on unsupplemented minimal medium. Suppressed and unsuppressed $t d_{201}$ mutants were grown on minimal medium supplemented with indole or tryptophan. All cultures were harvested after 72 to $96 \mathrm{hr}$ incubation. The $t d+s u_{201}$ carboys were inoculated with ascospore cultures to insure the presence of the suppressor. Harvesting and lyophilization of mycelia, preparation of crude extracts, and purification of normal tryptophan synthetase (TSase) and mutationally altered tryptophan synthetase (CRM) to the $\mathbf{R}_{25-31}$ stage (about 15-20 percent pure) were performed as previously described (MoHLER and Suskind 1960).

Artificial $\mathbf{R}_{25-31}$ mixtures of wild-type and $t d_{201}$ TSase, in proportions designed to simulate TSase activity ratios in suppressed $t d_{201}$ mutants, were prepared either by mixing $\mathbf{R}_{25-31}$ preparations of the two enzymes (Reconstitute ${ }_{a}$ ) or by fractionation of mixed mycelia to the $R_{25-31}$ stage (Reconstitute ${ }_{b}$ ). About $3 \mathrm{ml}$ of $\mathrm{R}_{25-31}$ fractions containing approximately $50 \mathrm{mg}$ protein per $\mathrm{ml}$ were further purified on a $2 \times 170 \mathrm{~cm}$ G-100 Sephadex column prepared as described (Pharmacia Brochure 1961) at room temperature with a solution A eluant (see Heat inactivation). Fractions of $3 \mathrm{ml}$ were collected in a refrigerated fraction collector. G-100 fractions of maximum specific activity were used for acrylamide gel electrophoresis, Ouchterlony agar diffusion, and sucrose density gradient centrifugation.

Assay procedures: TSase reactions 1, 2, and $3 \mathrm{f}$ were assayed as described by Yourvo and Suskind (1964). Bovine serum albumin $(0.8 \mathrm{mg} / \mathrm{ml})$ was added to $R_{25-31}$ or G-100 preparation assay mixtures to stabilize the enzyme. Protein in crude extracts and $R_{25-31}$ fractions was determined by the microbiuret assay (Yourno and Suskind 1964), and in the G-100 samples by the optical density at $280 \mathrm{~m} \mu$.

Physical, biochemical, and immunological characterization of TSase and CRM prepared from $\mathrm{su}^{+}{ }_{201}$ and $\mathrm{su}_{201}$ genotypes: Ratio of Reaction 2 enzyme to antigen in crude extracts of $\mathrm{td}^{+} \mathrm{su}^{+}{ }_{201}$ and $\mathrm{td}+\mathrm{su}_{201}$ strains: The ratio of enzyme/antigen in crude extracts was determined according to published methods (Suskind 1957). Fresh crude extracts were prepared from young mycelia (grown in $200 \mathrm{ml}$ aerated cultures at $30^{\circ} \mathrm{C}$ ) to minimize loss of enzymatic relative to antigenic activity. Inocula for $t d+s u_{201}$ cultures were taken directly from ascospore cultures.

Heats of activation of reaction 1: $\mathrm{R}_{25-31}$ preparations were diluted to 5-7 units of reaction 1 per $\mathrm{ml}$ in solution A containing $1 \mathrm{mg}$ BSA per ml, and reaction 1 activity was assayed at $17^{\circ}$, $28^{\circ}$ and $37^{\circ} \mathrm{C}$. The initial velocity is expressed as $\mu$ moles indoleglycerolphosphate (InGP) disappearing per $\mathrm{hr}$ per $\mathrm{ml}$ extract. The activation energy for each preparation was calculated from the slope of the Arrhenius plot.

$K_{m}$ for indoleglycerolphosphate in Reaction $3 f: \mathrm{K}_{\mathrm{m}}$ for $\mathrm{InGP}$ in reaction $3 \mathrm{f}$ was determined with $\mathbf{R}_{25-31}$ TSase diluted in $\mathrm{pH} 6.2$ buffer to approximately 1 unit reaction $3 \mathrm{f} \mathrm{per} \mathrm{ml}$. The reaction mixture contained $0.1 \mathrm{ml}$ enzyme, $260 \mu$ moles $\mathrm{pH} 6.2$ phosphate buffer, $0.2 \mu$ moles pyridoxal phosphate, $1 \mathrm{mg}$ BSA, $2 \times 10^{-3} \mathrm{M}$ EDTA and 0.04 to $0.80 \mu$ moles InGP in a final volume of $1.0 \mathrm{ml}$. In order to insure a linear rate of indole formation the reaction was stopped before reaching 10 percent completion.

$p H$ optimum of reaction $3 f: \mathrm{R}_{25-31}$ samples were diluted in $\mathrm{pH} 6.2$ buffer to approximately 1 unit of reaction $3 \mathrm{f}$ activity per $\mathrm{ml}$. The reaction mixture consisted of $0.1 \mathrm{ml}$ enzyme in solution A, $250 \mu$ moles phosphate buffer at the $\mathrm{pH}$ indicated, $1 \mathrm{mg}$ BSA, $0.2 \mu$ moles InGP, $0.2 \mu$ moles pyridoxal-P and $6 \times 10^{-3} \mathrm{M}$ EDTA in a final volume of $0.9 \mathrm{ml}$.

Heat inactivation of TS ase and $\mathrm{td}_{201} C R M: \mathrm{R}_{25-31}$ preparations were diluted to $2 \mathrm{mg}$ protein per $\mathrm{ml}$ in solution $\mathrm{A}, \mathrm{X}$ or $\mathrm{Z}$. Solution $\mathrm{A}$ contained $10^{-3} \mathrm{M}$ EDTA, $0.12 \mu$ moles pyridoxal-P and $100 \mu$ moles phosphate buffer, $\mathrm{pH} 7.8$, per ml. Solutions $\mathrm{Z}$ and $\mathrm{X}$ were identical to solution A except that solution $\mathrm{Z}$ contained only $0.014 \mu$ moles pyridoxal-P per $\mathrm{ml}$ while solution $\mathrm{X}$ contained $0.5 \mu$ moles pyridoxal-P and $10 \mu$ moles DL-serine per $\mathrm{ml} .0 .5 \mathrm{ml}$ aliquots were preheated 
at $37^{\circ} \mathrm{C}$ for 3 to $5 \mathrm{~min}$, then placed in a water bath at the appropriate temperature and swirled for the first 30 seconds. At specified times the tubes were plunged in ice water, swirled for 30 sec, and stored in an icebath until assayed.

Gel filtration: Sephadex gels were prepared as described (Pharmacia brochures 1961, 1962) and $1 \times 20 \mathrm{~cm}$ columns were used. $t d_{201} s u_{201-8}$ or $1: 1$ mixtures of wild type and $t d_{201} \mathbf{R}_{25-31}$ preparations ( 8 to $16 \mathrm{mg}$ protein) were eluted at room temperature with either $0.1 \mathrm{~m}$ phosphate buffer $\mathrm{pH} 6.2$ containing $10^{-3} \mathrm{M}$ EDTA; a stepwise gradient consisting of $0.1 \mathrm{M}$ phosphate buffer $\mathrm{pH} 6.2$ containing $10^{-3} \mathrm{M}$ EDTA and $0.5 \mathrm{~m}$ phosphate buffer $\mathrm{pH} 6.2$ containing $5 \times 10^{-3} \mathrm{M}$ EDTA; or a linear gradient of $0.1 \mathrm{~m}$ to $0.9 \mathrm{M}$ phosphate buffer $\mathrm{pH} 7.8$ containing $10^{-3} \mathrm{M}$ EDTA. In all experiments fractions of approximately $1.5 \mathrm{ml}$ were collected.

Ouchterlony agar double diffusion: As a criterion of enzyme purity agar diffusion experiments were performed as described (Oudin 1952). The center well in each experiment contained approximately 100 units of rabbit anti-TSase prepared against partially purified TSase (strain C-84). TSase preparations from G-100 columns were added to the outer wells in successive dilutions.

Acrylamide gel electrophoresis of TSase and CRM: Preparation of acrylamide gels and electrophoresis buffer, $\mathrm{pH}$ 9.6, application of samples on filter paper strips, and staining for protein were performed as described (Smati, Harrington and KeILley 1961). Samples from G-100 columns each containing 100-150 $\mu$ g protein, were run for $31 / 2$ hours at $6 \mathrm{v} / \mathrm{cm}$.

Sucrose density gradient centrifugation: The procedure for measuring the molecular weight of an enzyme by determining the ratio of the sedimentation velocity of an unknown to a standard enzyme was followed (MARTIN and Ames 1961). The $4.55 \mathrm{ml}$ linear gradients of 5 to 20 percent sucrose in $0.1 \mathrm{~m}$ phosphate buffer, $\mathrm{pH} 7.8$, containing $10^{-3} \mathrm{M}$ EDTA were prepared in $1.3 \times 5.1$ cm cellulose nitrate tubes. A $0.075 \mathrm{ml}$ aliquot of a Sephadex G-100 fraction of TSase containing 0.11 to $0.15 \mathrm{mg}$ protein and $0.025 \mathrm{ml}$ of the catalase standard ( $\mathrm{mol} \mathrm{wt} \mathrm{250,000)}$ containing $0.03 \mathrm{mg}$ protein were carefully layered on the sucrose gradient and centrifuged $13 \mathrm{hr}$ at 38,000 $\mathrm{rev} / \mathrm{min}$ in a refrigerated model L Spinco ultracentrifuge equipped with an SW-39 swinging bucket rotor. The bottom of each tube was punctured with a \#26 needle, the needle withdrawn, and $31 \pm 5$ fractions of two drops each were collected and assayed. Drop size was found to increase somewhat with fraction number because of the decrease in hydrostatic pressure during emptying of the tube. Correction factors for increase in drop size were determined for each tube after collection of the enzyme fractions by refilling the tube with an identical sucrose gradient containing Bromphenol blue and plotting increase in absorbancy at $600 \mathrm{~m} \mu$ with fraction number after collection of two drop fractions in a standard volume of distilled water. Molecular weight values calculated with the correction factor, however, did not change by more than \pm 5 percent.

\section{RESULTS}

Suppressed $t d_{201}$ mutants were found to retain the high level of reaction 2 activity of TSase characteristic of the unsuppressed mutant. In addition they possess about 10 to 20 percent of the wild-type level of TSase reaction 1 activity. While reaction $3 \mathrm{f}$ activity cannot be detected in crude extracts of suppressed mutants, it is measurable in fractionated preparations.

Biochemical and physical studies of mutant, suppressed mutant, and wild-type TSase: Mixing experiments with crude extracts and deproteinized supernatants of crude extracts of $t d_{201}$ and $t d_{201} s u_{201-8}$ strains showed that inhibitor or activator type relationships cannot be the basis of recovered reaction 1 activity in $t d_{201}$ $s u_{201-8}$. No inhibition of $t d_{201} s u_{201-8}$ reaction 1 activity by $t d_{201}$ extracts, nor evocation of this activity in $t d_{201}$ CRM by $t d_{201} s u_{201-8}$ extracts was observed. Furthermore $t d_{201} s u^{+}{ }_{201-8} \mathrm{CRM}$ does not acquire reactions 1 or $3 \mathrm{f}$ activity, nor do $t d_{201} s u_{201-8}$ or $t d_{201} s u_{201-2}$ extracts lose reaction 1 activity on fractionation. 
$\mathrm{R}_{25-31}$ preparation of $t d_{201} s u_{201-8}$ and $t d_{201} s u_{201-2}$ and Sephadex G-100 preparations of $t d_{201} s u_{201-2}$ contain reaction $3 \mathrm{f}$ activity in a ratio to reaction 1 activity approximating that of wild-type strains, i.e., 1 to 50.

Characterization of recovered activities of suppressed mutants: Various physical and enzymatic characteristics of the TSase activities in $R_{25-31}$ preparations of $t d_{201} s u_{201-8}$ and $t d_{201} s u_{201-2}$ were compared to those of wild-type enzyme as well as to those of artificial mixtures of $t d_{201}$ and wild-type enzyme mixed in proportions of TSase activities simulating those exhibited by the suppressed mutant enzyme.

Energy of activation of reaction 1: TSase reaction 1 activity of suppressed mutants possesses an energy of activation similar to that of wild type and of artificial mixtures of $t d_{201}$ and wild-type TSase, viz., 13,000 to $14,000 \mathrm{cal} / \mathrm{mole} \mathrm{InGp} \mathrm{in}$ the temperature range $17^{\circ}$ to $37^{\circ} \mathrm{C}$ (Figure 1).

$K_{\mathrm{m}}$ for InGP and $p H$ optimum of Reaction $3 f$ : Reaction $3 \mathrm{f}$ activity of suppressed mutant TSase, like that of artificial mixtures, has a wild-type $K_{m}$ value for InGP of 2.5 to $3.0 \times 10^{-4} \mathrm{M}$ (Figure 2) and exhibits a wild-type $\mathrm{pH}$ optimum of 6.2 to 6.4 (Figure 3).

Heat stability: Reaction 2 activity of suppressed mutant TSase was found to be far more heat stable than reactions 1 or $3 f$ in phosphate buffer containing pyridoxal-P. The kinetics of loss of all three TSase activities are slightly greater than first order, but while reaction 1 and $3 \mathrm{f}$ exhibit a half-life of about 100 seconds at $60^{\circ} \mathrm{C}$, reaction 2 has a half-life of approximately 25 minutes (Table 1). Under

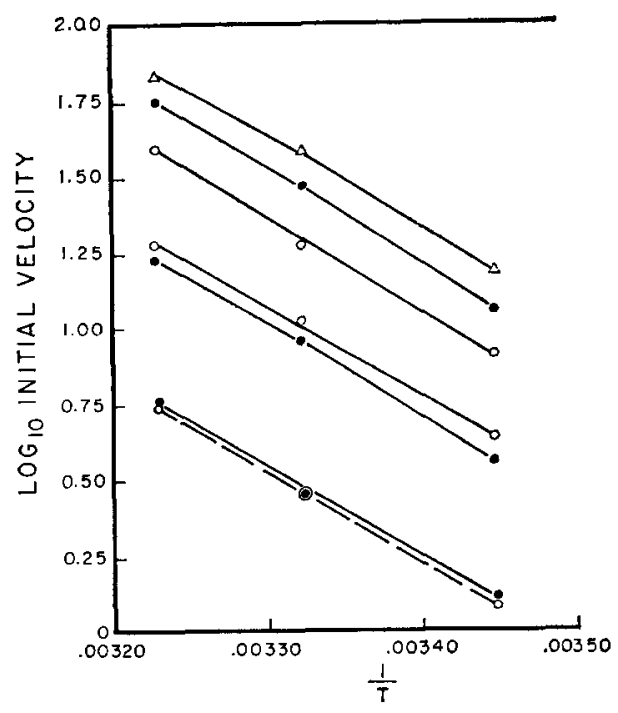

FIGURE 1.-Arrhenius plot of reaction 1 activity of $R_{25-31}$ TSase from $s u^{+}{ }_{201}$ and $s u_{201}$ genotypes. The activation energies, calculated from the slopes and expressed as $\mathrm{cal} / \mathrm{mole} \mathrm{InGP}$ are, from top to bottom: $t d{ }_{201-2}, 13,300 ; t d+s u{ }^{+201-8}, 14,200 ; t d+s u_{201-8}, 13,900$; Reconstitute a $\left(t d_{201} s u{ }^{201-8}+t d+s u{ }_{201-8}\right), 13,100 ; t d_{201} s u_{201-2}, 13,800 ; t d_{201} s u_{201-8}, 13,400$; Reconstitute $\left(t d_{201} s u+{ }_{201-8}+t d+s u{ }_{201-8}\right), 13,600$. 


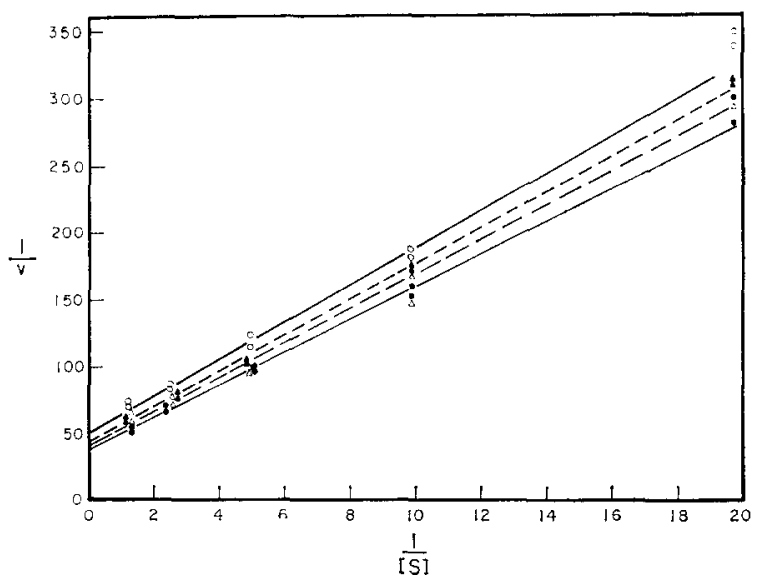

Frgure 2.- $\mathrm{K}_{\mathrm{m}}$ for InGP, reaction 3f, of $\mathrm{R}_{25-31}$ TSase from $s{ }^{+}{ }_{201}$ and $s u_{201}$ genotypes. O $t d_{201} s u_{201-8}, 2.8 \times 10^{-4} \mathrm{M} ; \Delta$ Reconstitute ${ }_{\mathrm{b}}\left(t d_{201} s u{ }_{201-8}+t d{ }^{+} s u{ }^{+201-8}, 3.0 \times 10^{-4} \mathrm{M}\right.$; $\triangle t d{ }^{s u+}{ }_{201-8}, 3.1 \times 10^{-4} \mathrm{M} ; \bigcirc t d+s u_{201-8}, 3.1 \times 10^{-4} \mathrm{M}$. Preparations of $t d_{201} s u_{201-2}$ and $t d+s u_{201-2}$ TSase exhibited similar values, viz., $3.1 \times 10^{-4}$ and $2.7 \times 10^{-4} \mathrm{M}$ respectively. $\mathrm{S}$ represents the molar concentration of InGP and $\mathrm{v}$ the initial reaction velocity.

the same conditions $t d_{201}$ CRM reaction 2 activity declines in a manner similar to that of suppressed mutant TSase reaction 2 , both with respect to half-life and reaction order. In contrast, all three wild-type Tsase activities are inactivated initially with first-order kinetics and have a half-life of approximately 60 seconds at $60^{\circ} \mathrm{C}$. Reconstituted mixtures of $t d_{201}$ and wild-type TSase behave as would be predicted from the properties of the individual components.

Wild-type TSase is protected from heat inactivation in the presence of both

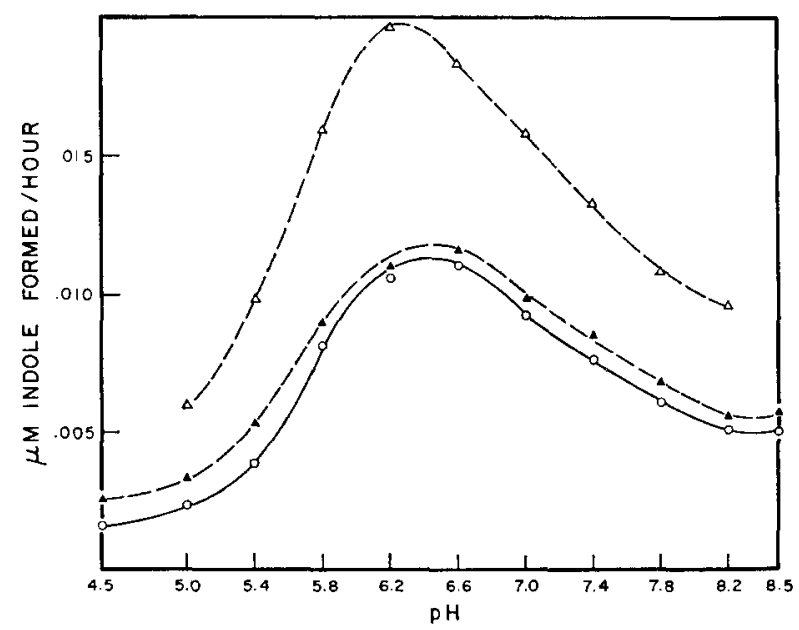

Figure 3.-pH optimum, reaction 3f, of $\mathrm{R}_{25-31}$ TSase from $s u+{ }_{201}$ and $s u_{201}$ genotypes. $\triangle t d{ }^{+} s u^{+}{ }_{201-8} ; \Delta$ Reconstitute ${ }_{\mathrm{b}}\left(t d_{201} s u^{+}{ }_{201-8}+t d^{+} s{ }^{+}{ }_{201-8}\right) ; O t d_{201} s u_{201-8} \cdot t d_{201} s u_{201-2}$ TSase gave similar results. 
TABLE 1

Heat inactivation of $R_{25-31}$ preparations of TSase or CRM from su+ ${ }_{201}$ and $\mathrm{su}_{201}$ genotypes

\begin{tabular}{|c|c|c|c|c|c|c|c|c|}
\hline \multirow[b]{3}{*}{ Enzyme } & \multicolumn{6}{|c|}{ Inactivation at $60^{\circ} \mathrm{C}$ in phosphate-pyridoxal-P buffer } & \multirow{3}{*}{\multicolumn{2}{|c|}{$\begin{array}{c}\text { Increase in } \mathrm{T}_{1 / 2} \text { at } \\
58^{\circ} \mathrm{C} \text { in presence of } \\
\text { pyridoxal- } \mathrm{P} \text { and serine } \\
\text { Reaction }\end{array}$}} \\
\hline & \multicolumn{3}{|c|}{$\mathrm{T}_{1 / 2}$ in seconds } & \multicolumn{3}{|c|}{ Reaction order } & & \\
\hline & 1 & $\underset{2}{2}$ & $3 \mathrm{f}$ & 1 & $\underset{2}{\operatorname{Reactio}}$ & $3 f$ & & \\
\hline$t d+s u+{ }_{201-8}$ & 60 & 52 & 63 & $1^{\circ}$ & $1^{\circ}$ & $1^{\circ}$ & 4.6 & 4.6 \\
\hline$t d+s u_{201-8}$ & 64 & 52 & 63 & $1^{\circ}$ & $1^{\circ}$ & $1^{\circ}$ & $\ldots$ & . \\
\hline$t d_{201} s u^{+}{ }_{20 t-8}$ & & 1200 & & & $>1^{\circ}$ & & & 1.1 \\
\hline $\begin{array}{l}t d_{201} s u_{201-8} \\
\text { Reconstitute } \\
(t d+s u+\end{array}$ & 120 & 1400 & 90 & $>1^{\circ}$ & $>1^{\circ}$ & $>1^{\circ}$ & 5.6 & 1.2 \\
\hline$\left.t d_{201} s u+{ }_{201-8}\right)$ & 56 & 1200 & 56 & $1^{\circ}$ & $>1^{\circ}$ & $1^{\circ}$ & 4.6 & 1.2 \\
\hline
\end{tabular}

Reaction oxder and half-life of activity loss of $R_{25-81}$ TSase or CRM was determined from semilog plots of heat inacti. vation data. $t d_{201} s u_{201-2}$ and $t d^{+} s u_{201-2}$ enzyme preparations were inactivated like those of $t d_{201} s u_{201-8}$ and $t d^{+} s u_{201-8}$ respectively. Inactivation of Reconstitute, was similar to that of Reconstitute ${ }_{b}$.

DL-serine and pyridoxal-P (Mohrer and Suskind 1960). At $58^{\circ} \mathrm{C}$ the half-life of both reactions 1 and 2 of wild-type TSase is increased approximately fivefold by addition of these compounds while reaction 2 of $t d_{201}$ CRM is not significantly protected. Under the same conditions the half-life of reaction 1 of suppressed mutant TSase is increased approximately fivefold while the half-life of reaction 2 is increased no more than 10 to 20 percent. Reconstituted mixtures of $t d_{201}$ CRM and wild-type enzyme are indistinguishable from suppressed mutant TSase in this respect (Table 1).

Sephadex gel filtration: Attempts were made to separate $t d_{201}$ CRM and wildtype enzyme in artificial mixtures (1:1 by activity) and in suppressed mutant extracts by column chromatography of $R_{25-31}$ fractions through a variety of ionexchange and neutral Sephadex gels. Passage of a $t d_{201} s u_{201-8}$ preparation through a short DEAE-Sephadex (A-50) column failed to separate reactions 1 and 2. Neither did the artificial mixtures show detectable skewing of reactions 2 and $3 f$ on short DEAE-Sephadex (A-50 or A-25), Sulfoether-Sephadex (SE-50), or neutral Sephadex (G-200) columns. Furthermore, large scale preparative fractionation of mutant, suppressed mutant, and wild-type $R_{25-31}$ TSase preparations on Sephadex G-100 columns indicated similar behavior for all the samples. Specific activity peaks for reactions 1 and 2 of suppressed mutant TSase also coincided by this fractionation method.

Sucrose density gradient centrifugation: Physical characterization of the enzymes and further attempts at separation of mutant and wild-type species were performed with highly purified G-100 preparations. On Ouchterlony agar double diffusion the mutant and suppressed mutant enzyme preparations gave a single precipitin band when tested with anti-wild-type TSase while the wild-type enzyme preparation showed a weak second band as well.

Mutant, suppressed mutant, and wild-type enzyme were found to have similar 
molecular weights as determined by sucrose density gradient centrifugation. The approximate value of 110,000 to $120,000 \mathrm{~mol}$ wt obtained with sucrose gradient centrifugation is in reasonable agreement with the value previously obtained by sedimentation and diffusion measurements (Mohler and Suskind 1960; CARsiotis, Appella, and Suskind 1964; Ensign, Kaplan, and Bonner 1964). Reaction 1 activity of suppressed mutant TSase sedimented coincidentally with reaction 2. As was expected by the similar fractionation behavior of mutant and wild-type enzyme, the reaction 1 activity of a small amount of wild-type TSase added to the $t d_{201}$ CRM preparation did not separate from $t d_{201}$ reaction 2 activity. This was confirmed by the uniform sedimentation of a 1:1:1:1 mixture of $t d^{+} s u^{+}{ }_{201}, t d^{+} s u_{201}, t d_{201} s u_{201}$, and $t d_{201} s u^{+}{ }_{201}$ preparations. Single and coincidental reaction 1 and reaction 2 peaks were obtained which showed no spread relative to the urmixed control enzyme profiles.

Acrylamide gel electrophoresis: Aliquots of these G-100 enzyme preparations were compared by acrylamide gel electrophoresis. Again no evidence of a physical difference between mutant, suppressed mutant, and wild-type enzymes was obtained. No additional component, representing a putative second enzyme species in addition to $t d_{201}$ CRM, was detected by electrophoresis of suppressed mutant samples.

Effect of $\operatorname{td}_{201}$ suppressors on the wild-type phenotype: To assess the phenotypic effect of $t d_{201}$ suppressor genes on wild-type strains a number of enzymic, immunological and nutritional properties of $t d^{+} s u_{201}$ and $t d^{+} s u^{+}{ }_{201}$ strains were compared. The presence of the $t d_{201}$ suppressor in the culture from which $t d^{+} s u_{201-2}$ TSase was prepared was confirmed by appropriate crosses of the harvested mycelia.

Biochemical and physical characterization of $\mathrm{td}^{+} \mathrm{su}_{201}$ TSase: $\mathrm{R}_{25-31}$ preparations of $t d^{+} s u_{201-8}$ and $t d^{+} s u_{201-2}$ TSase were similar to the TSase of the $t d^{+} s u^{+}{ }_{201-s}$ control when judged by the $\mathrm{Km}$ for InGP in reaction 3f, energy of activation of reaction 1 , heat stability of all three TSase activities in solution $A$, and ratios of TSase activities (Figures 1, 2; Table 1).

Several additional properties of suppressed and unsuppressed wild-type enzymes were examined. The purification patterns of enzymes from two $t d^{+} s u^{+}{ }_{21^{-}}$ $t d^{+} s u_{201}$ pairs were similar at the $\mathrm{R}_{25-31}$ stage both with respect to degree of purification and recovery of enzyme activity. The $t d^{+} s u^{+}{ }_{201-2}$ and $t d^{+} s u_{201-2} \mathrm{R}_{25-31}$ TSase preparations also fractionate similarly on Sephadex G-100 columns. Suppressed wild-type enzyme, like the unsuppressed, has a molecular weight of 110,000 to 120,000 as determined by sucrose density gradient centrifugation and is indistinguishable from the wild type on acrylamide gel electrophoresis. Like the $t d^{+} s u^{+}{ }_{201-2}$ enzyme, the $t d^{+} s u_{201-2}$ G-100 TSase preparation used for these studies showed a very weak second precipitin band against anti-wild-type TSase in Ouchterlony agar double diffusion.

Immunological characterization of $\mathrm{td}^{+} \mathrm{su}_{201}$ TSase: The ratio of reaction 2 enzyme activity to antigen in crude extracts of young mycelia is close to unity for two $t d^{+} s u_{201}-t d^{+} s u^{+}{ }_{201}$ pairs originating in the same tetrad. No antigenic 
TABLE 2

Comparison of ratios of enzyme/antigen in crude extracts of young mycelia of $\mathrm{su}_{201}$ and $\mathrm{su}_{201}$ genotypes

\begin{tabular}{lcc}
\hline \multicolumn{1}{c}{ Strain } & enzyme/antigen \\
\hline$t d+s u{ }^{201-8}$ & $(2$ experiments $)$ & $1.0 \pm 0.1$ \\
$t d+s u_{201-8}$ & & $1.0 \pm 0.1$ \\
$t d+s u{ }_{201-2}$ & $(2$ experiments $)$ & $1.0 \pm 0.1$ \\
$t d{ }^{+s u_{201-2}}$ & & $1.0 \pm 0.1$ \\
$t d_{201} s u{ }^{201-8}$ & $(2$ experiments $)$ & $0.7 \pm 0.05$ \\
$t d_{201} s u_{201-8}$ & & $0.7 \pm 0.05$ \\
\hline
\end{tabular}

The ratio of enzyme/antigen with respect to reaction 2 was determined for each crude extract as described in METHons. Each experiment was performed with extracts prepared from different cultures.

difference was observed between crude extract TSase of $t d_{201} s u^{+}{ }_{201-8}$ and $t d_{201}$ $s u_{201-8}$ strains, both types exhibiting a ratio of reaction 2 enzyme activity to antigen of approximately 0.7 (Table 2 ).

Nutritional characteristics of $\mathrm{td}^{+} \mathrm{su}_{201}$ strains: The only consistent difference between $t d^{+} s u_{201}$ and $t d^{+} s u^{+}{ }_{201}$ strains from the same tetrad involved a slight decrease in the initial growth rate of $t d^{+} s u_{201}$ on minimal medium (Figure 4). Nevertheless each $t d^{+} s u_{201}-t d^{+} s u^{+}{ }_{201}$ pair attained comparable final growth levels and in all cases proved equally inhibitable by DL-tryptophan, although growth of the $t d^{+} s u_{201}$ strains was less stimulated by complete medium (Table 3 ).

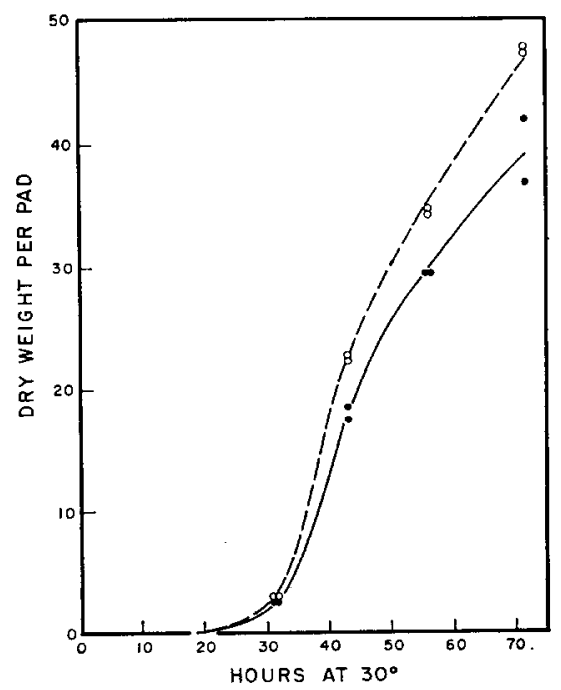

FIGURE 4.-Comparison of minimal medium growth rates of $t d+s u+{ }_{201}$ and $t d+s u_{201}$ strains. $\bigcirc t d+s{ }^{\circ}{ }_{201-2} ; \bigcirc t d+s u_{201-2}, t d+$ strains carrying either $s u_{201-4}, s u_{201-6}, s u_{201-7}$, or $s u_{201-8}$ exhibited a similar decrease in growth rate relative to $t d{ }^{+} s u+{ }_{201}$ controls originating in the same tetrad. For each $t d+s u+{ }_{201}-t d{ }^{+} s u_{201}$ pair examined, inocula were adjusted to equal concentrations of viable conidia. Dry weight per pad is expressed in mg. 
TABLE 3

Comparison of the growth of $\mathrm{td}+\mathrm{su}+{ }_{201}$ and $\mathrm{td}+\mathrm{su}_{201}$ genotypes originating in the same tetrad

\begin{tabular}{|c|c|c|c|}
\hline Strain & $\begin{array}{l}\text { Dry weight (mg) } \\
\text { of terminal culture } \\
\text { in minimal medium }\end{array}$ & $\begin{array}{c}\text { Effect of } 150 \mu \mathrm{g} \\
\text { DL-tryptophan } \\
\text { per ml on log } \\
\text { phase growth rate* }\end{array}$ & $\begin{array}{l}\text { Effect of complete } \\
\text { medium on log } \\
\text { phase growth rate }\end{array}$ \\
\hline$t d+s u^{+}{ }_{201-2}$ & 159 & 83 & 160 \\
\hline$t d+s u_{201-2}$ & 150 & 63 & 108 \\
\hline$t d+s{ }^{+}{ }_{201-6}$ & 139 & 66 & 181 \\
\hline$t d+s u_{201-6}$ & 150 & 70 & 149 \\
\hline$t d+s u+{ }_{201.7}$ & 146 & 60 & 160 \\
\hline$t d+s u_{201-\tau}$ & 146 & 55 & 113 \\
\hline$t d+s u{ }_{201-4}$ & 147 & 64 & 195 \\
\hline$t d+s u_{201-4}$ & 141 & 57 & 70 \\
\hline$t d+s u^{+}{ }_{201-8}$ & 96 & & . \\
\hline$t d+s u_{201-8}$ & 104 & & \\
\hline
\end{tabular}

* Expressed as percent rate in minimal medium.

\section{DISCUSSION}

It was shown by mixing experiments and by the fractionation behavior of mutant and suppressed mutant enzymes that the low level of activities of reactions 1 and $3 \mathrm{f}$ in suppressed $t d_{201}$ mutants does not appear to reflect activation or inhibition phenomena. Rather, the suppressor mutation seems to be responsible for restoring normal structure to a fraction of the enzyme molecules. The implication, by analogy to the studies of suppressor action in the $E$. coli TSase system (BRoDY and YANOFSKY 1963), is that the original wild-type amino acid has been reinserted, although alternatively, a second residue change in each CRM molecule might have occurred (Yanofsky, Henning, Helinski and Carlton 1963), resulting in a partially regained capacity of the CRM to catalyze reactions 1 and 3f. Unfortunately, attempts at physical separation of mixtures of $t d_{201}$ and wildtype enzyme or of the putative two enzyme species in suppressed mutant extracts were unsuccessful, so that we are unable to distinguish between these possibilities.

Reversion studies with the E. coli alkaline phosphatase (GAREN 1960) and Neurospora TSase (Esser, DEMoss, and BonNER 1960) systems suggest that all revertants with normal enzymatic characteristics are primary site revertants. In contrast, revertants with aberrant enzymatic characteristics test either as primary or as second site revertants. Fingerprint studies of revertant enzymes in the E. coli TSase A protein system (Allen and Yanofsky 1963; Henning and Yanofsky 1962; Yanofsky, Henning, Helinski and Carlton 1963) indicate that revertants with enzymatically normal A protein contain the wild-type or an equally functional amino acid reinserted at the primary mutant site in the polypeptide chain. Enzymatically abnormal revertant $A$ proteins contain either a second mutant amino acid at a site distinct from that of the primary mutant site, 
or a different amino acid than the wild-type residue at the primary mutant site.

In this light, it seems most reasonable to suggest that the low levels of reactions 1 and $3 f$ activities in suppressed $t d_{201}$ mutants indicate the formation of a small amount of wild-type or wild-type-like TSase together with characteristic levels of CRM. The enzymological properties of the suppressed enzyme, i.e., InGP utilizing activities, are so similar to those of the wild-type protein that secondsite reversion or the insertion of an amino acid less functional than the wild-type at the primary site seems unlikely. The slightly greater than wild-type half-life of heat inactivation observed for suppressed mutant TSase reactions 1 and $3 f$ could reflect the presence of a functional amino acid different from the wild-type residue or to in vivo association of mutant and wild-type subunits (CARsiotrs, Appella and Suskind 1964).

The properties of a suppressed $t d_{201}$ TSase have also been examined by RAcHMELER and ST. LAwRENCE (1964) who conclude the active enzyme is physically more similar to the mutant CRM. It appears that the site of this $t d_{201}$ suppressor mutation is not identical with ours (ST. LAWRENCE, personal communication).

In considering the mode of action of $t d_{201}$ suppressors an unrestricted "mistake" mechanism, whether at the level of the adaptor system (Y YNoFsky and ST. Lawrence 1960; Yanofsky, Helinski and Maling 1961) or the ribosome (Davies, Gilbert and Gorini 1964; Gorini and Kataja 1964), might be expected to substitute one specific amino acid for another in all cell proteins, thereby producing considerable quantities of altered proteins.

Thus the lack of gross changes in the enzymatic and nutritional characteristics of suppressed strains found in this study seems more compatible with a "lowlevel" mistake mechanism of suppressor action, or one involving a switch of similar amino acid residues.

A "nonsense to sense" mechanism of $t d_{201}$ suppressor action does not appear as plausible. It appears (Crick, Barnetr, Brenner and Watts-Tobin 1961; SaraBhai, Stretton, Brenner and Bolle 1964) that the sequential synthesis of the polypeptide chain may stop at the nonsense triplet. Therefore, mutants of this type very likely would be CRM-negative (Suskind, WickHam and Carsiotis 1963; Kaplan, Ensign, Bonner and Mills 1964). The $t d_{201}$ mutant site is known to be near the middle of the $t d$ locus (Kaplan, Surama and Bonner 1964). If this site corresponds to an approximate midpoint in the reading frame of the gene and the $t d_{201}$ suppressors act by a nonsense-to-sense mechanism, then some mechanism for reading past the nonsense triplet in $t d_{201}$ must exist, since $t d_{201}$ forms CRM of normal molecular weight.

To recapitulate, $t d_{201}$ suppressors were found to have little if any effect on $t d^{+}$ genotypes (or on $t d_{201}$ genotypes aside from restoring some wild-type TSase activity). In consonance with these findings, Rachmeler and St. Lawrence (1964) could detect no changes in gross amino acid composition of suppressed $t d_{201}$ or suppressed wild-type strains. A slight inhibition of growth was observed when the suppressor was present in a wild-type strain, and this was not relieved by any components of the complete medium. Similar results have been reported previously for a suppressed TSase mutant (YANofsky 1952). A site-specific sup- 
pressor of an inositolless mutant of Neurospora also appears to exert an inhibitory effect on the wild-type (Giles and Partridge 1953). On the other hand, sitespecific suppressors of $E$. coli alkaline phosphatase (GAREN and Siddror 1962) and Salmonella typhimurium purine-requiring mutants (Gots 1956) do not detectably alter the growth of wild types. Indeed, a site-specific suppressor of isoleucineless mutants of Saccharomyces cerevisiae has been reported to stimulate the growth of the wild type on minimal medium, or of the mutant grown in the presence of isoleucine (KaKar 1963). One limitation of most of these growth tests is the unavailability of isogenic stocks for comparison. Although growth comparisons were made here only between $t d^{+} s u^{+}{ }_{201}-t d^{+} s u_{201}$ pairs originating in the same tetrad, no claim for isogenicity can be made. Hence the growth effect may be due to genes other than $s u_{201}$.

\section{SUMIMARY}

Enzymological and physical studies of TSase from suppressed $t d_{201}$ mutants suggest the presence of wild-type enzyme as well as CRM. When the $t d_{201}$ suppressors are crossed into wild-type strains there is no observable change in the enzymic, antigenic, or physical properties of wild-type TSase. Nevertheless a slight decrease in growth rate does occur on either minimal or complete medium. These data are considered in the context of current theories of suppressor gene action in protein synthesis.

\section{LITERATURE CITED}

Allen, M. K., and C. Yanofsky, 1963 A biochemical and genetic study of reversion within the $A$ gene. Genetics $48: 1065-1083$.

Benzer. S., and S. Crrampe, 1962 A change from nonsense to sense in the genetic code. Proc. Natl. Acad. Sci. U.S. 48 : 1114-1121.

BRody, S., and C. YANofsky, 1963 Suppressor gene alteration of protein primary structure, Proc. Natl. Acad. Sci. U.S. 50: 9-16.

Carsiotis. M., E. Appella, and S. R. Suskind, 1964 Chemical and physical studies of the structure of tryptophan synthetase from Neurospora crassa. (Manuscript in preparation.)

Crick. F. H. C., L. Barnett, S. Brenner, and R. J. Watts-Tobin, 1961 General nature of the genetic code for proteins. Nature 192: 1227-1232.

Davies, J., W. Gilbert, and L. Gorini, 1964 Streptomycin, suppression and the code. Proc. Natl. Acad. Sci. U.S. 51 : 883-890.

Ensign, S., S. Kaplan, and D. M. Bonner, 1964 Purification and partial characterization of tryptophan synthetase from Neurospora crassa. Biochim. Biophys. Acta 81 : 357-366.

Esser, K., J. A. DeMoss, and D. M. Bonner, 1960 Reverse mutations and enzyme heterogeneity. Z. Vererb. 91 : 291-299.

GAREN, A., 1960 Genetic control of the specificity of the bacterial enzyme, alkaline phosphatase. Symp. Soc. Gen. Microbiol. 10: 239-247.

GAREN, A., and O. Siddiqr, 1962 Suppression of mutations in the alkaline phosphatase structural cistron of E. coli. Proc. Natl. Acad. Sci. U.S. 48: 1121-1126.

Giles, N. H.. and C. Partridge, 1953 The effect of a suppressor on allelic inositolless mutants in N. crassa. Proc. Natl. Acad. Sci. U.S. 39: 479-488. 
Gorini, L., and E. KataJA, 1964 Phenotypic repair by streptomycin of defective genotypes in E. coli. Proc. Natl. Acad. Sci. U.S. 51 : 487-493.

Gots, J., 1956 The biochemical nature of a suppressor mutation in a purine-requiring mutant of Salmonella typhimurium. Carnegie Inst. Wash. Publ. 612: 77-86.

HeNning, U., and C. Yanofskr, 1962 Amino acid replacements associated with reversion and recombination within the $A$ gene. Proc. Natl. Acad. Sci. U.S. 48: 1497-1504.

KAKAR, S. N., 1963 Suppressor mutations for the isoleucine locus in Saccharomyces. Genetics 48: $967-979$.

Kaplan, S., S. Ensign, D. M. Bonner, and S. E. Mrlls, 1964 Gene products of CRM- mutants at the $t d$ locus. Proc. Natl. Acad. Sci. U.S. 51 : 372-378.

Kaplan, S., Y. SuYama, and D. M. BonNer, 1964 Fine structure analysis at the $t d$ locus of Neurospora crassa. Genetics 49: 145-158.

Martin, R. G., and B. N. AmEs, 1961 A method of determining the sedimentation behavior of enzymes: application to protein mixtures. J. Biol. Chem. 236: 1372-1379.

Mohler, W. C., and S. R. Suskind, 1960 The similar properties of tryptophan synthetase and a mutationally altered protein in Neurospora crassa. Biochim. Biophys. Acta 43: 288-299.

OudiN, J., 1952 Specific precipitation in gels and its application to immunological analysis. Methods Med. Res. 5 : 335-378.

Pharmacia Brochure on Neutral Sephadex. 1961.

Pharmacia Brochures on DEAE- and SE-Sephadex. 1962.

Rachmeler, M., and P. St. Lawrence, 1964 Suppressor gene effects in tryptophan synthetase mutants. (Abstr.) Fed. Proc. 23 : 378.

Ryan, F. J., 1950 Selected methods of Neurospora genetics. Methods Med. Res. 3 : 51 -75.

Sarabhai, A., A. Stretton, S. Brenner, and A. Bolle, 1964. Colinearity of the gene with the polypeptide chain. Nature 201: 13-17.

Smald, P. A., W. F. Harrington, and W. W. Keilley, 1961 The electrophoretic homogeneity of myosin subunits. Biochim. Biophys. Acta 49 : 462-470.

SuskrNd, S. R., 1957 Properties of a protein antigenically related to tryptophan synthetase in Neurospora crassa. J. Bacteriol. 74 : 308-318.

Suskind, S. R., M. L. WickhaM, and M. Carsiotis, 1963 Anti-enzymes in immunogenetic studies. Ann. N.Y. Acad. Sci. 103 : 1106-1127.

YANofsky, C., 1952 The effects of gene changes on tryptophan desmolase formation. Proc. Natl. Acad. Sci. U.S. 38: 215-226.

YANOFsky, C., and I. Crawford, 1959 The formation of a new enzymatically active protein as a result of suppression. Proc. Natl. Acad. Sci. U.S. 45: 1280-1287.

Yanofsky, C., and P. St. Lawrence, 1960 Gene action. Ann. Rev. Microbiol. 14: 311-340.

Yanofsky, C., D. Helinski, and B. Maling, 1961 The effects of mutation on the composition and the properties of the A protein of Escherichia coli tryptophan synthetase. Cold Spring Harbor Symp. Quant. Biol. 26: 11-23.

Yanofsky, C., U. Henning, D. Herrnsiki, and B. Carlton, 1963 Mutational alteration of protein structure. Fed. Proc. 22 : 75-79.

Younno, J., and S. R. Suskind, 1963 Wild-type-like enzyme in a suppressed tryptophan synthetase mutant of Neurospora. (Abst.) Genetics 48: 917-918. _ $\quad 1964$ Suppressor gene action in the tryptophan synthetase system of Neurospora crassa. I. Genetic studies. Genetics 50: $803-816$. 